\title{
Can indoor navigation service incorporating signs support spatial learning?
}

\author{
Wangshu Wang ${ }^{\mathrm{a},}$, Haosheng Huang ${ }^{\mathrm{b}}$, Georg Gartner ${ }^{\mathrm{a}}$ \\ ${ }^{a}$ Research Division Cartography, Department of Geodesy and Geoinformation, Vienna University of Technology, Vienna, Austria, \\ wangshu.wang@tuwien.ac.at,georg.gartner@tuwien.ac.at \\ ${ }^{b}$ Department of Geography, Ghent University, Ghent, Belgium, haosheng.huang@ugent.be \\ * Corresponding author
}

Keywords: Indoor Navigation, Signage, Spatial Knowledge Acquisition, Location Based Services

\begin{abstract}
:
Spatial information is a necessity for successful navigation. While in familiar environments, we can rely on prior acquired spatial knowledge, in unfamiliar or partially familiar environments, we highly depend on external information to successfully reach destinations. Navigation services are therefore designed to navigate users from an origin to a destination. However, empirical studies suggest that current navigation systems, which guide users with turn-by-turn instructions, negatively affect users' spatial learning in the short term (Gardony et al. 2013, Parush et al. 2007), as well as spatial ability in the long term (Ishikawa 2019). To compensate for the negative influence, researchers (Brügger et al. 2017, Waters \& Winter 2011) proposed actively engaging the users with the environment, by incorporating landmarks into navigation services.
\end{abstract}

Attempts have been made to classify indoor landmarks and integrate them into navigation services (Ohm et al. 2014, Feller et al. 2017), but due to the nature of the indoor environment, identifying indoor landmarks are still more difficult than in outdoors. As a subset of semantic landmarks, directional signs are meant to facilitate navigation by presenting immediate navigation information (Gibson 2009). Therefore, we proposed to incorporate directional signs into indoor navigation service in a previous study (Wang et al. 2019), attempting to direct users' attention back to the environment and assist their spatial learning.

In this study, we conducted an in-situ experiment to examine the viability of the route instructions generated by the aforementioned indoor navigation service, guided by the following research questions:

1. Is the route instructions generated by the proposed indoor navigation service effective? How is its performance, in terms of effectiveness and efficiency, compared with turn-by-turn intrusions?

2. How do the users perceive it in terms of preference?

3. Can it support users' spatial knowledge acquisition?

Our experiment consisted of two route following tasks, each with a set of subsequent spatial knowledge assessment tasks, and ended with a questionnaire. The two routes are similar in length in the route following tasks, and both include some level change. On one route, the participant was guided by the route instructions generated by the proposed indoor navigation service. On the other route, the participant was guided by turn-by-turn instructions. The combination of the routes and the instructions were randomised. The spatial knowledge assessment tasks include direction estimates, landmark recognition, ordering, and placement on the floor plan, etc. As we recruited students and conducted the experiment in a university building, the questionnaire featured their familiarity with the building and their experience with the route guidance.

To avoid potential interruptions caused by localisation inaccuracy indoors, we implemented the experiment with the "Wizard of Oz" method (Kelley 1983). Inspired by Bakogiannis et al. (2019), we generated route instructions beforehand and translated them into successive images along the route (Figure 1). We then used two android smartphones. One was given to the participant, with an image switching app simulating an indoor navigation service. The other was held by an experimenter, controlling the participant's phone. There was a second experimenter, who recorded the route following process. 


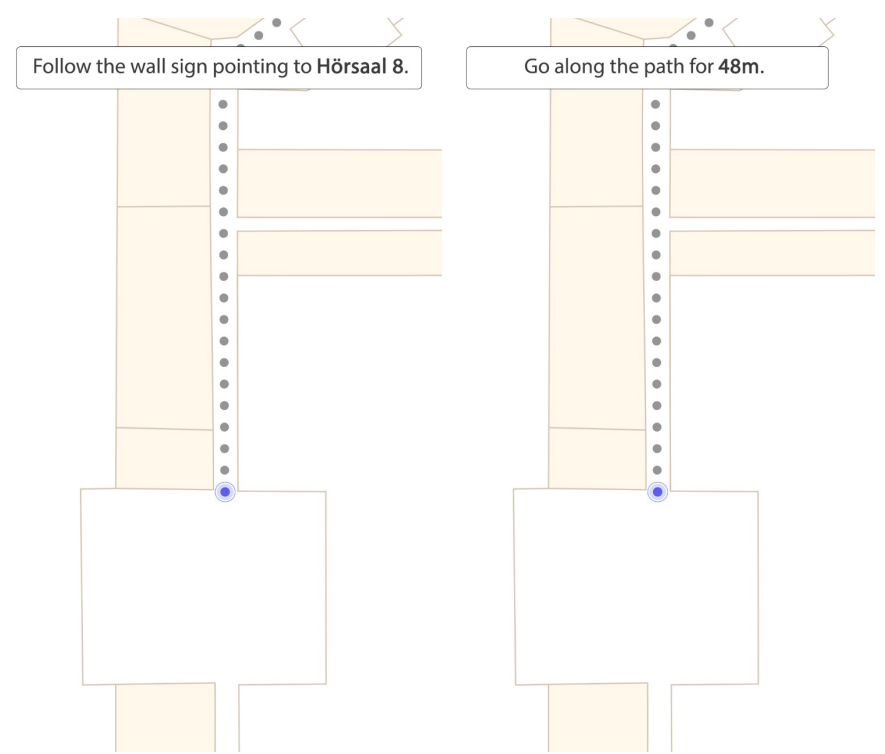

Figure 1. Images presenting the route instructions.

We will first screen the participants based on their familiarity. Then we plan to assess participant's performance by the number of stops and travel time. Their preference will be derived from the questionnaire. The spatial knowledge assessment tasks will inform us about their spatial knowledge acquisition during route following.

\section{References:}

Bakogiannis, N., Gkonos, C., \& Hurni, L. (2019). Cartographic Visualization for Indoor Semantic Wayfinding. Multimodal Technologies and Interaction, 3(1), 22. https://doi.org/10.3390/mti3010022

Brügger, A., Richter, K. F., \& Fabrikant, S. I. (2017). Distributing Attention Between Environment and Navigation System to Increase Spatial Knowledge Acquisition During Assisted Wayfinding. In International Conference on Spatial Information Theory (pp. 19-22). Springer, Cham

Fellner, I., Huang, H., \& Gartner, G. (2017). "Turn Left after the WC, and Use the Lift to Go to the 2nd Floor"Generation of Landmark-Based Route Instructions for Indoor Navigation. ISPRS International Journal of GeoInformation, 6(6), 183. https://doi.org/10.3390/ijgi6060183

Gardony, A. L., Brunyé, T. T., Mahoney, C. R., \& Taylor, H. A. (2013). How navigational aids impair spatial memory: Evidence for divided attention. Spatial Cognition \& Computation, 13(4), 319-350. https://doi.org/10.1080/13875868.2013.792821

Gibson, D. (2009). The wayfinding handbook: Information design for public places. Princeton Architectural Press.

Ishikawa, T. (2019). Satellite navigation and geospatial awareness: Long-term effects of using navigation tools on wayfinding and spatial orientation. The Professional Geographer, 71(2), 197-209. https://doi.org/10.1080/00330124.2018.1479970

Kelley, J. F. (1983, December). An empirical methodology for writing user-friendly natural language computer applications. In Proceedings of the SIGCHI conference on Human Factors in Computing Systems (pp. 193-196).

Ohm, C., Müller, M., Ludwig, B., \& Bienk, S. (2014). Where is the landmark? Eye tracking studies in large-scale indoor environments. In P. Kiefer, I. Giannopoulos, M. Raubal, \& A. Krüger (Eds.), Proceedings of the 2nd International Workshop on Eye Tracking for Spatial Research (Vol. i, pp. 47-51). Vienna, Austria.

Parush, A., Ahuvia, S., \& Erev, I. (2007). Degradation in spatial knowledge acquisition when using automatic navigation systems. In International Conference on Spatial Information Theory (pp. 238-254). Springer, Berlin, Heidelberg.

Wang, W., Huang, H., Lyu, H., \& Gartner, G. (2019). Incorporating directional signs into indoor navigation systems. Abstr. ICA, 1, 1-2.

Waters, W., \& Winter, S. (2011). A wayfinding aid to increase navigator independence. Journal of Spatial Information Science, 2011(3), 103-122. 